\title{
Inverse p-Median Problems with Variable Edge Lengths
}

Fahimeh Baroughi Bonab, Rainer Ernst Burkard, and Elisabeth Gassner

$$
\text { Project Area(s): }
$$

Effizient lösbare kombinatorische Optimierungsprobleme

Institut für Optimierung und Diskrete Mathematik (Math B) 


\title{
Inverse $p$-Median Problems with Variable Edge Lengths
}

Fahimeh Baroughi Bonab • Rainer E. Burkard · Elisabeth Gassner

Received: date / Accepted: date

\begin{abstract}
The inverse $p$-median problem with variable edge lengths on graphs is to modify the edge lengths at minimum total cost with respect to given modification bounds such that a prespecified set of $p$ vertices becomes a $p$ median with respect to the new edge lengths. The problem is shown to be strongly $\mathcal{N} \mathcal{P}$-hard on general graphs and weakly $\mathcal{N} \mathcal{P}$-hard on series-parallel graphs. Therefore, the special case on a tree is considered: It is shown that the inverse 2-median problem with variable edge lengths on trees is solvable in polynomial time. For the special case of a star graph we suggest a linear time algorithm.
\end{abstract}

Keywords Location problem · inverse optimization $\cdot p$-median $\cdot$ complexity analysis

\section{Introduction}

In recent years inverse optimization problems found an increasing interest. In an inverse optimization problem the task is to change parameters of the problem (like traffic connections or client rankings) at minimum cost so that a prespecified solution becomes optimal. In one of the first papers on this

F. Baroughi Bonab

Graz University of Technology, Institute of Optimization and Discrete Mathematics, Steyrergasse 30, 8010 Graz, Austria. E-mail: baroughi@opt.math.tugraz.at

Sahand University of Technology, Faculty of Basic Sciences for Engineering, Department of Applied Mathematics, Tabriz, Iran.

R.E. Burkard

Graz University of Technology, Institute of Optimization and Discrete Mathematics, Steyrergasse 30, 8010 Graz, Austria. E-mail: burkard@opt.math.tugraz.at

E. Gassner

Graz University of Technology, Institute of Optimization and Discrete Mathematics, Steyrergasse 30, 8010 Graz, Austria. E-mail: Gassner@opt.math.tugraz.at 
subject, Burton and Toint [8] investigated the inverse shortest path problem. It is beyond of the scope of this paper to give a complete overview about inverse optimization problems. For a survey on inverse optimization problems the reader is referred to Heuberger [16].

In the following, we will restrict ourselves to inverse location problems. In classical location problems the aim is to locate one or more facilities such that the (weighted) distances from clients to facilities is smallest possible. Hence, the quality of facility locations depends on weights of clients and on the distances between clients and facilities. Two well-known location problems are the $p$-median and the $p$-center location model: While in the $p$-median problem the goal is to find a set of $p$ facilities such that the sum of (weighted) distances from the clients to the closest facility becomes minimum, the $p$-center location problem is to determine a set of $p$ facilities such that the maximum (weighted) distance between clients and the closest facility is minimized. Most attention was paid to location problems on graphs and in the plane. For location problems in graphs the positions of clients coincide with the set of vertices. The distance between two points is measured by the shortest distance in the graph with respect to given edge lengths. In case of location problems in the plane, the distance is usually measured by the $L_{1^{-}}, L_{2^{-}}$or $L_{\infty}$-norm. Hence, if we consider an inverse approach then the parameters that can be modified are client weights and/or edge lengths (in case of a problem on a graph) or client weights and/or positions (in case of a problem in the plane).

Let us start with inverse median problems in graphs: In 2004, Burkard, Pleschiutschnig and Zhang [6] considered inverse $p$-median problems with variable vertex weights and showed that discrete inverse $p$-median problems can be solved in polynomial time, if $p$ is fixed and not an input parameter. Moreover, the same authors $[6,7]$ investigated the inverse 1-median problem with variable vertex weights and proved that the problem is solvable by a greedytype algorithm in $O(n \log n)$ time if the underlying network is a tree or the location problem is defined in the plane (where distances are measured by the rectilinear or the Chebyshev norm) and in $O\left(n^{2}\right)$ time on cycles. On the other hand, Gassner [10] proved that the inverse 1-maxian problem with variable edge lengths on general graphs is strongly $\mathcal{N} \mathcal{P}$-hard and remains weakly $\mathcal{N} \mathcal{P}$ hard even on series-parallel graphs. The author suggested an $O(n \log n)$ time algorithm for this problem on a tree. An inverse version of the convex ordered median problem was studied by Gassner [11]. The author showed that this problem is $\mathcal{N} \mathcal{P}$-hard even on trees. Further, it was shown that the problem remains $\mathcal{N} \mathcal{P}$-hard for unit weights or if the underlying problem is a $k$-centrum problem (but not, if both of these conditions hold). The inverse unit-weight $k$ centrum problem with unit cost coefficients on a tree can be solved in $O\left(n^{3} k^{2}\right)$ time. Let us now consider inverse median problems in the plane. Observe that the 1-median problem in the plane with respect to the Euclidean distance is called Fermat-Weber problem. In 2008, Burkard, Galavii and Gassner [5] suggested a Greedy-type algorithm for the inverse Fermat-Weber problem with variable vertex weights, unit cost and under the assumption that the prespecified point that should become a 1-median does not coincide with a given 
weighted point in the plane. The inverse 1-median problem with variable coordinates is to modify the positions (= coordinates) of the given points (= clients) in $\mathbb{R}^{d}$ at minimum cost such that a prespecified point in $\mathbb{R}^{d}$ becomes a 1-median. The inverse 1-median problem with variable coordinates was investigated by Baroughi, Burkard and Alizadeh [3]. They showed that this problem in $\mathbb{R}^{d}$ endowed with the rectilinear norm is $\mathcal{N} \mathcal{P}$-hard, but it can be solved by a pseudo-polynomial algorithm. It was shown that the problem is polynomially solvable when the point weights are assumed to be equal. The authors suggested an $O(d n)$ time algorithm for the problem in $\mathbb{R}^{d}$ endowed with the squared Euclidean norm. Moreover, it was shown that the problem in the plane endowed with the Chebyshev norm is $\mathcal{N} \mathcal{P}$-hard. They proposed another pseudo-polynomial algorithm for this case.

Let us now turn our attention to inverse center problems. Cai, Yang and Zhang [9] proved that the inverse 1-center location problem with variable edge lengths on general unweighted graphs is $\mathcal{N} \mathcal{P}$-hard, while the underlying center location problem is solvable in polynomial time. Yang and Zhang [22] considered the inverse vertex center problem with variable edge lengths on tree networks. They derived an $O\left(n^{2} \log n\right)$ time algorithm for this problem where it is assumed that all the modified edge lengths remain positive. Alizadeh and Burkard [1] investigated the inverse absolute and vertex 1-center location problem on trees with variable edge lengths. They showed that the absolute and vertex 1-center problem can be solved in $O\left(n^{2}\right)$ time provided that all edge lengths are strictly positive. Dropping this condition, they proposed an $O\left(n^{2} r\right)$ time algorithm where the parameter $r$ bounded by $\left\lceil\frac{n}{2}\right\rceil$. Recently Alizadeh, Burkard and Pferschy [2] used a set of suitably extended AVL-search trees and developed a combinatorial algorithm which solves the inverse 1-center location problem with edge length augmentation in $O(n \log n)$ time. In this article we investigate the inverse $p$-median problem with variable edge lengths.

The article is organized as follows: In the next section, we state the inverse $p$-median problem on general graphs. Afterwards, Section 3 concerns some complexity results. We prove that the inverse $p$-median problem is strongly $\mathcal{N} \mathcal{P}$-hard and remains weakly $\mathcal{N} \mathcal{P}$-hard on series-parallel graphs. In Section 4 we recall the edge deletion method for finding a 2-median of a tree. Using this method we can formulate the inverse 2-median problem on a tree as a linear program which is solvable in polynomial time. Finally, in Section 5 we show that the inverse 2-median problem on star graphs can be solved in linear time by using the special structure of the linear programming formulation of Section 4 combined with the fast multidimensional search algorithm of Megiddo [19].

\section{The $p$-median problem and the inverse $p$-median problem with variable edge lengths}

Let $G=(V, E)$ be a connected graph with vertex set $V=\left\{v_{1}, \ldots, v_{n}\right\}$, edge set $E$ with $|E|=m$, and a constant $p \leq n$. Every edge $e \in E$ has a positive 
length $\ell_{e}$ and $d_{\ell}\left(v_{i}, v_{j}\right)$ denotes the shortest distance from $v_{i} \in V$ to $v_{j} \in V$ in $G$ with respect to length vector $\ell$. Moreover, for any vertex $v \in V$ let $w(v)$ be a positive vertex weight. In the classical p-median problem the goal is to locate $p$ pairwise different facilities $m_{1}, \ldots, m_{p}$ on $G$ (i.e., on vertices or edges) which minimize the sum of weighted distances from each vertex to its closest facility:

$$
\min _{m_{1}, \ldots, m_{p} \in G} \sum_{v \in V} w(v) \min _{i=1, \ldots, p} d_{\ell}\left(v, m_{i}\right)
$$

An optimal solution is called $p$-median.

Hakimi [15] showed that there exists an optimal solution among the set of vertices. This property is called vertex optimality.

The classical $p$-median problem has been studied since the 60's. Kariv and Hakimi [18] showed that this problem is $\mathcal{N} \mathcal{P}$-hard even if $G=(V, E)$ is a planar graph of maximum degree 3 . In the case of tree graphs the $p$-median problem is solvable in polynomial time. Kariv and Hakimi [18] developed an algorithm that computes a solution in $O\left(p^{2} n^{2}\right)$ time. The running time was improved to $O\left(p n^{2}\right)$ by Tamir [21] and later to $O\left(n \log ^{p+2} n\right)$ by Benkoczi and Bhattacharya [4]. For the 1-median problem on trees algorithms that run in linear time were given by Hua et al. [17] and independently by Goldman [13]. The case of $p=2$ can be solved in $O(n \log n)$ time as shown by Gavish and Sridhar [14].

Now we are going to define the inverse $p$-median problem with variable edge lengths: Let a connected graph $G=(V, E)$ with a positive weight $w(v)$ for every $v \in V$ and a positive length $\ell_{e}$ for every $e \in E$ be given. We want to modify the edge lengths at minimum cost such that a given set of vertices $\left\{m_{1}, \ldots, m_{p}\right\}$ becomes a $p$-median. Suppose that we incur nonnegative cost $c_{e}^{+}$, if $\ell_{e}$ is increased by one unit and we incur nonnegative $\operatorname{cost} c_{e}^{-}$, if $\ell_{e}$ is decreased by one unit. Let $p_{e}$ and $q_{e}$ be the amounts by which the edge length $\ell_{e}$ is increased and decreased, respectively. We suppose that $p_{e}$ and $q_{e}$ obey the upper bounds $u_{e}^{+}$and $u_{e}^{-}$. Moreover, let $\mathcal{S}$ denote the set of all subsets $S \subseteq V$ of cardinality $|S|=p$. Therefore, we can state the inverse $p$-median problem on graph $G$ as follows:

Modify the edge lengths $\ell_{e}, e \in E$, to $\tilde{\ell}_{e}=\ell_{e}+p_{e}-q_{e}$ such that the following three properties hold:

(i) The set $\left\{m_{1}, \ldots, m_{p}\right\}$ becomes a $p$-median of $G$ with respect to $\tilde{\ell}$, i.e.,

$$
\sum_{v \in V} w(v) \min _{i=1, \ldots, p} d_{\tilde{\ell}}\left(v, m_{i}\right) \leq \sum_{v \in V} w(v) \min _{k \in S} d_{\tilde{\ell}}\left(v, v_{k}\right) \quad \text { for all } S \in \mathcal{S} .
$$

(ii) The bound constraints are satisfied:

$$
0 \leq p_{e} \leq u_{e}^{+}, \quad 0 \leq q_{e} \leq u_{e}^{-} \quad \text { for all } e \in E
$$


(iii) The linear cost function

$$
\sum_{e \in E}\left(c_{e}^{+} p_{e}+c_{e}^{-} q_{e}\right)
$$

becomes minimum.

This formulation of the inverse $p$-median problem is a nonlinear programming model. In Section 4, we will, however, get a linear programming formulation of this problem if the underlying graph is a tree.

In the next section we are going to investigate the computational complexity of the inverse $p$-median problem on general and also on series-parallel graphs. It requires first to discuss the complexity of the special case $p=1$.

\section{Computational complexity of inverse $p$-median problems}

In this section, we first show that the inverse 1-median problem is strongly $\mathcal{N} \mathcal{P}$ hard on general graphs. Then we generalize this result to $p$-median problems for any $p \in \mathbb{N}$. Next we show that the inverse 1 -median problem and also the inverse $p$-median problem remain weakly $\mathcal{N} \mathcal{P}$-hard on series-parallel graphs. Our $\mathcal{N} \mathcal{P}$-hardness constructions are motivated by the $\mathcal{N} \mathcal{P}$-hardness proof for the inverse 1-maxian problem by Gassner [10]. Since the ideas are similar to those in [10], we will omit some details and refer the interested reader to the paper by Gassner.

3.1 Strong $\mathcal{N} \mathcal{P}$-hardness of the inverse 1-median problem on general graphs

We consider an instance of the Set Cover problem which is known to be strongly $\mathcal{N} \mathcal{P}$-hard [12], to construct an equivalent instance of the inverse 1median problem on general graphs.

An instance $I_{S C}$ of the Set Cover problem is given by a finite set $\mathcal{S}$, a family $\mathcal{C}$ of subsets and an integer $K<|\mathcal{C}|$. The Set Cover problem asks for a set of subsets $\mathcal{C}^{\prime} \subseteq \mathcal{C}$ of cardinality at most $K$ such that every element of $\mathcal{S}$ belongs to at least one set in $\mathcal{C}^{\prime}$.

Let us assume that we are given an instance $I_{S C}$. Now we construct an instance $I_{I 1 M}$ of the inverse 1-median problem with a graph $G=(V, E)$ in the following way:

$$
\begin{aligned}
V & =\{s, t\} \cup\left\{a_{i} \mid i \in \mathcal{S}\right\} \cup\left\{v_{j} \mid C_{j} \in \mathcal{C}\right\} \\
E & =E_{1} \cup E_{2} \cup E_{3} \\
E_{1} & =\left\{\left(s, a_{i}\right) \mid i \in \mathcal{S}\right\} \\
E_{2} & =\left\{\left(a_{i}, v_{j}\right) \mid i \in \mathcal{S}, C_{j} \in \mathcal{C}, i \in C_{j}\right\} \\
E_{3} & =\left\{\left(v_{j}, t\right) \mid C_{j} \in \mathcal{C}\right\}
\end{aligned}
$$

The vertex weights are defined in the following way: $w\left(a_{i}\right)=1$ for every $i \in \mathcal{S}$ and $w(x)=0$ otherwise. All edges $e \in E$ have equal length $\ell_{e}=1$. Only 


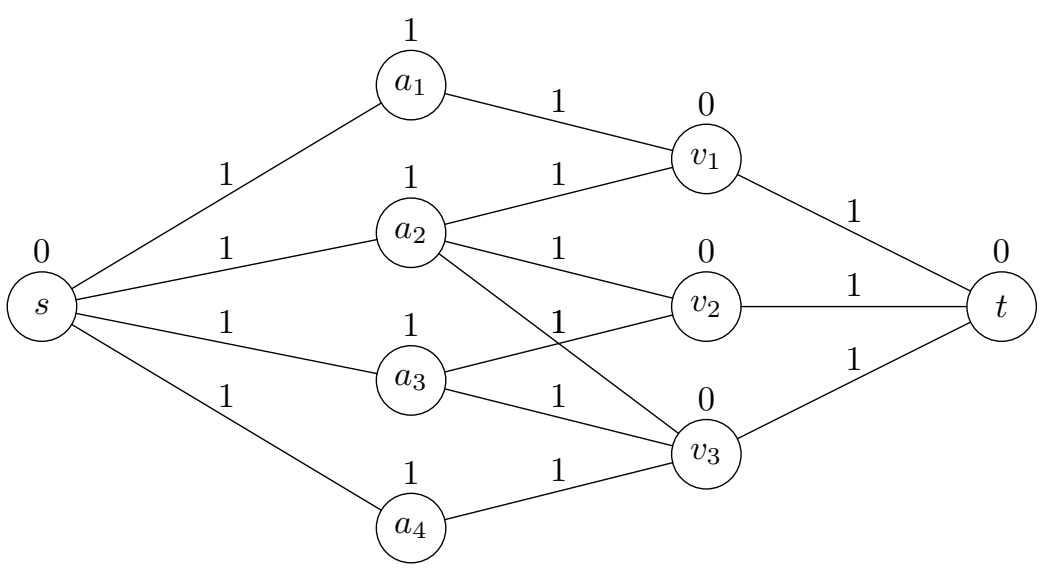

Fig. 1 Graph constructed from the Set Cover instance with $\mathcal{S}=\{1,2,3,4\}$ and $\mathcal{C}=$ $\{\{1,2\},\{2,3\},\{2,3,4\}\}, \mathcal{C}^{\prime}=\{\{1,2\},\{2,3,4\}\}$

$E_{3}$ has edges whose lengths are allowed to be modified. We define $u_{e}^{-}=1$ if $e \in E_{3}$ and $u_{e}^{-}=0$ otherwise. Finally, $u_{e}^{+}=0$ for all $e \in E$. We also assume that $c_{e}^{+}=c_{e}^{-}=1$ for all $e \in E$. The definition of the instance of the inverse 1median problem is completed by requiring that vertex $t$ becomes a 1-median. A specific example for the constructed instance in this way is illustrated in Figure 1.

Let $(p, q)$ be any length modification that satisfies the bound constraints and let $\ell=\ell+p-q$. Then the objective function values of the vertices of $G$ with respect to $\tilde{\ell}$ fulfill the following properties:

$$
\begin{aligned}
f_{\tilde{\ell}}(s)=|\mathcal{S}| & & f_{\tilde{\ell}}\left(a_{i}\right)=2(|\mathcal{S}|-1)>|\mathcal{S}| & i \in \mathcal{S} \\
f_{\tilde{\ell}}(t) \geq|\mathcal{S}| & & f_{\tilde{\ell}}\left(v_{j}\right) \geq|\mathcal{S}| & C_{j} \in \mathcal{C}
\end{aligned}
$$

Therefore, we conclude that $t$ is a 1 -median with respect to $\tilde{\ell}$ if and only if $f_{\tilde{\ell}}(t)=|\mathcal{S}|$.

Lemma 1 There exists a Set Cover of cardinality at most $K$ for $I_{S C}$ if and only if there exists a feasible solution of the constructed instance of the inverse 1 -median problem of cost at most $K$.

Proof This lemma can be proved in an analogous way as Lemma 2.1 in [10].

Since the Set Cover problem is strongly $\mathcal{N} \mathcal{P}$-hard we obtain the following theorem.

Theorem 1 The inverse 1-median problem with variable edge lengths is strongly $\mathcal{N P}$-hard on general graphs even if all lengths and cost coefficients are equal to 1 . 
3.2 Strong $\mathcal{N} \mathcal{P}$-hardness of the inverse $p$-median problem on general graphs

The hardness of the inverse 1-median problem can be generalized to the inverse $p$-median problem for any natural number $p$.

Given an instance $I_{I 1 M}$ with $G=(V, E)$, weights $w(v)(v \in V)$, lengths $\ell_{e}(e \in E)$, cost coefficients $c_{e}^{+}, c_{e}^{-}$, and bounds $u_{e}^{+}, u_{e}^{-}$and vertex $m_{p}$ which should become a 1-median. We construct an instance $I_{I P M}$ of the inverse $p$-median problem on a graph $G^{\prime}=\left(V^{\prime}, E^{\prime}\right)$ as follows:

$$
\begin{aligned}
& V^{\prime}=V \cup V_{1} \\
& V_{1}=\left\{m_{1}, \ldots, m_{p-1}\right\} \\
& E^{\prime}=E \cup E_{1} \\
& E_{1}=\left\{\left(m_{i}, m_{i+1}\right) \mid i=1, \ldots, p-1\right\}
\end{aligned}
$$

All vertices $v \in V$ keep their vertex weights and all edges $e \in E$ maintain their lengths, cost coefficients and bounds as in $I_{I 1 M}$. It remains to define these parameters for elements in $V_{1}$ and $E_{1}$ : Their weights and lengths are chosen sufficiently large, i.e., $w(m)=M=\sum_{v \in V} w(v)+1$ for every vertex $m \in V_{1}$ and $\ell_{e}=L=\sum_{e \in E}\left(\ell_{e}+u_{e}^{+}\right)+1$ for every $e \in E_{1}$. Moreover, let $u_{e}^{+}=u_{e}^{-}=0$ and $c_{e}^{+}=c_{e}^{-}=1$ for each $e \in E_{1}$. Finally, the set $\left\{m_{1}, \ldots, m_{p-1}, m_{p}\right\}$ should become a $p$-median.

Let $\hat{\ell}$ be any length modification of $I_{I P M}$ that satisfies the bound constraints. We show that any $p$-median of graph $G^{\prime}$ with respect to $\hat{\ell}$ contains the vertices $m_{1}, \ldots, m_{p-1}$ :

Let $v_{j} \in V^{\prime}$ for $j=1, \ldots, p$ be arbitrary $p$ vertices of $V^{\prime}$ such that $m_{i} \neq v_{j}$ for $j=1, \ldots, p$ and every $m_{i} \in V_{1}$. Then we have

$$
f_{\hat{\ell}}\left(v_{1}, \ldots, v_{p}\right) \geq w\left(m_{i}\right) \min _{j=1, \ldots, p} d_{\hat{\ell}}\left(m_{i}, v_{j}\right) \geq M L .
$$

On the other hand, for every $v, v^{\prime} \in V$ we have

$$
d_{\hat{\ell}}\left(v, v^{\prime}\right) \leq \sum_{e \in E}\left(\ell_{e}+u_{e}^{+}\right)<L
$$

Let $m^{*} \in V$ be an arbitrary vertex, then

$$
\begin{aligned}
\frac{f_{\hat{\ell}}\left(m_{1}, \ldots, m_{p-1}, m^{*}\right)}{l} & =\sum_{v \in V^{\prime}} w(v) \min \left\{\min _{1 \leq j \leq p-1} d_{\hat{\ell}}\left(v, m_{j}\right), d_{\hat{\ell}}\left(v, m^{*}\right)\right\} \\
& =\sum_{v \in V} w(v) \min \{\min _{1 \leq j \leq p-1} \underbrace{d_{\hat{\ell}}\left(v, m_{j}\right)}_{\geq L}, \underbrace{d_{\hat{\ell}}\left(v, m^{*}\right)}_{<L}\} \\
& =\sum_{v \in V} w(v) d_{\hat{\ell}}\left(v, m^{*}\right)<M L \leq f_{\hat{\ell}}\left(v_{1}, \ldots, v_{p}\right) .
\end{aligned}
$$

Therefore, we conclude that every $p$-median of graph $G^{\prime}$ contains the vertices $m_{1}, \ldots, m_{p-1}$ and no vertex in $G$ travels to $m_{1}, \ldots, m_{p-1}$. Observe that 
any length modification $\hat{\ell}$ in $G^{\prime}$ implies a length modification $\tilde{\ell}$ in $G$ because the lengths of edges in $E_{1}$ are not allowed to be modified. Hence, let $\left\{m_{1}, \ldots, m_{p-1}, m^{*}\right\}$ with $m^{*} \in V$ be a candidate for a $p$-median in $G^{\prime}$. Then its objective value is equal to the 1-median objective value of $m^{*}$ in $G$. Moreover, we get $f_{\hat{\ell}}\left(m_{1}, \ldots, m_{p-1}, m^{*}\right)=f_{\tilde{\ell}}\left(m^{*}\right)$.

These observations immediately imply that $\left\{m_{1}, \ldots, m_{p-1}, m^{*}\right\}$ is a $p$ median of $G^{\prime}$ with respect to $\hat{\ell}$ if and only if $m^{*}$ is the 1-median of $G$ with respect to $\tilde{\ell}$. This leads to the following theorem.

Theorem 2 The inverse $p$-median problem with variable edge lengths is strongly $\mathcal{N} \mathcal{P}$-hard on general graphs for every $p \in \mathbb{N}$ even if all cost coefficients are equal to 1.

3.3 Weak $\mathcal{N} \mathcal{P}$-hardness of the inverse $p$-median problem on series-parallel graphs

In this subsection we first prove that the inverse 1-median problem is $\mathcal{N} \mathcal{P}$ hard even on series-parallel graphs. Next we show that the hardness of this problem can be generalized to the inverse $p$-median problem. Let us start with an $\mathcal{N} \mathcal{P}$-hardness proof of the inverse 1-median problem.

We prove by a reduction from the continuous multiple choice knapsack problem that the inverse 1-median is weakly $\mathcal{N} \mathcal{P}$-hard on series-parallel graphs. The continuous multiple choice knapsack problem is known to be weakly $\mathcal{N} \mathcal{P}$ hard, but it can be solve by a pseudo-polynomial algorithm [12].

An instance $I_{K P}$ of the continuous multiple choice knapsack problem is given by a finite set $\mathcal{S}$, for each $i \in \mathcal{S}$ a profit $b_{i} \in \mathbb{Z}^{+}$and a cost $c_{i} \in \mathbb{Z}^{+}$, a partition $\mathcal{A}$ of $\mathcal{S}$ into disjoint sets $A_{1}, \ldots, A_{k}$, and positive integers $P$ and $C$. The question is whether there exists a choice of a single element $\varphi(j) \in A_{j}$, $1 \leq j \leq k$, and an assignment of rational numbers $r_{j}, 0 \leq r_{j} \leq 1$, to the sets, such that

$$
\sum_{j=1}^{k} r_{j} c_{\varphi(j)} \leq C \quad \text { and } \quad \sum_{j=1}^{k} r_{j} b_{\varphi(j)} \geq P .
$$

Let us assume that we have an instance $I_{K P}$ of the continuous multiple choice knapsack problem. Now we construct an instance $I_{I 1 M}$ of the inverse 1-median problem on a series-parallel graph $G=(V, E)$ as follows.

$$
\begin{aligned}
V & =\{s, t\} \cup\left\{a_{j} \mid A_{j} \in \mathcal{A}\right\} \cup\left\{v_{i} \mid i \in \mathcal{S}\right\} \\
E & =E_{1} \cup E_{2} \cup E_{3} \\
E_{1} & =\left\{\left(s, a_{j}\right) \mid A_{j} \in \mathcal{A}\right\} \\
E_{2} & =\left\{\left(a_{j}, v_{i}\right) \mid i \in A_{j}, A_{j} \in \mathcal{A}\right\} \\
E_{3} & =\left\{\left(v_{i}, t\right) \mid i \in \mathcal{S}\right\}
\end{aligned}
$$




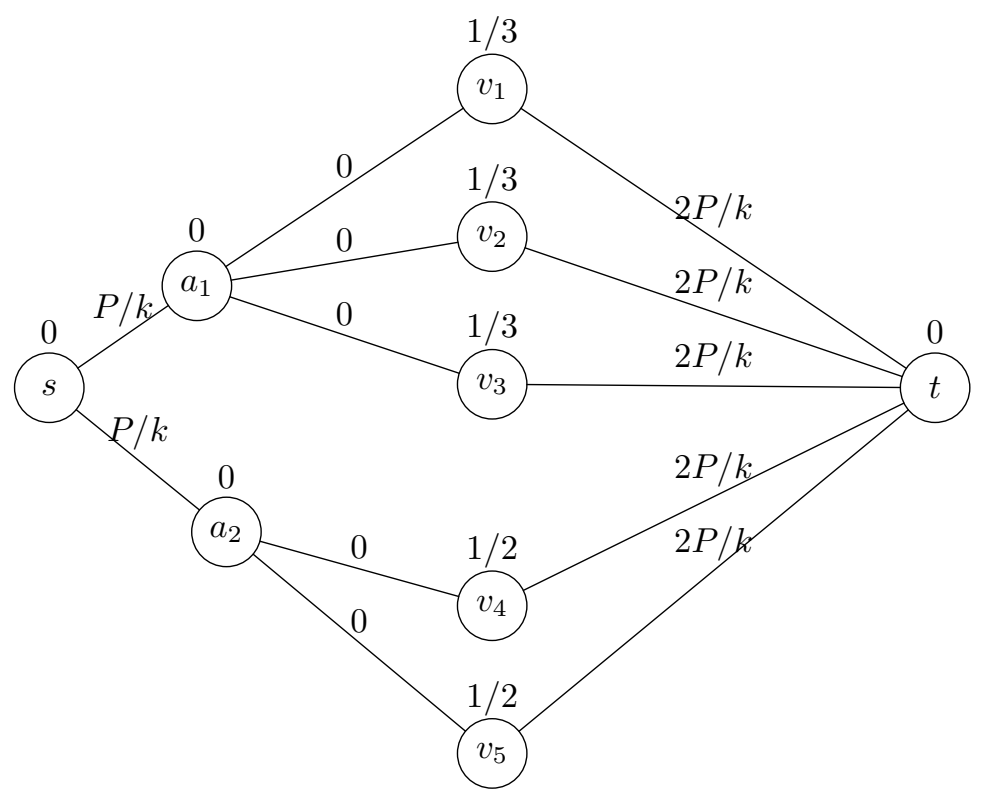

Fig. 2 Graph constructed from $\mathcal{A}=\left\{A_{1}, A_{2}\right\}$ and $A_{1}=\{1,2,3\}, A_{2}=\{4,5\}$.

The vertex weights are defined in the following way:

$$
w(x)= \begin{cases}\frac{1}{\left|A_{j}\right|} & \text { if } x=v_{i}, i \in A_{j}, \\ 0 & \text { otherwise. }\end{cases}
$$

We define length-, bound- and cost coefficients of the edges as follows.

$$
\ell_{e}=\left\{\begin{array}{ll}
\frac{P}{k} & \text { if } e \in E_{1} \\
0 & \text { if } e \in E_{2} \\
\frac{2 P}{k} & \text { if } e \in E_{3}
\end{array} \quad u_{e}^{-}=\left\{\begin{array}{ll}
b_{i} & \text { if } e \in E_{3} \\
0 & \text { otherwise }
\end{array} \quad c_{e}^{-}= \begin{cases}\frac{c_{i}}{b_{i}} & \text { if } e \in E_{3} \\
0 & \text { otherwise }\end{cases}\right.\right.
$$

Furthermore, $u_{e}^{+}=c_{e}^{+}=0$ for all $e \in E$.

The definition of an instance of the inverse 1-median problem is completed by specifying vertex $t$ as the vertex that should become a 1-median. Figure 2 illustrates the constructed instance for a specific example.

Observe, that edges in $E_{3}$ are only allowed to be shortened. Now we investigate the objective values of the vertices with respect to any feasible edge length modification: Let $(p, q)$ be any length modification that satisfies the bound constraints and let $\tilde{\ell}=\ell+p-q$. Then $f_{\tilde{\ell}}(s)=P, f_{\tilde{\ell}}\left(a_{j}\right)=f_{\tilde{\ell}}\left(v_{i}\right)$ for all $i \in A_{j}(j=1, \ldots, k)$ and

$$
f_{\tilde{\ell}}\left(a_{j}\right)=\sum_{\substack{i=1 \\ i \neq j}}^{k} d_{\tilde{\ell}}\left(a_{j}, a_{i}\right) \quad f_{\tilde{\ell}}(t)=\sum_{i=1}^{k} d_{\tilde{\ell}}\left(a_{i}, t\right)
$$


hold. Consider a vertex $a_{j}$ for $j \in\{1, \ldots, k\}$. Observe that

$$
d_{\tilde{\ell}}\left(a_{j}, a_{i}\right)=\min \left\{\frac{2 P}{k}, d_{\tilde{\ell}}\left(a_{j}, t\right)+d_{\tilde{\ell}}\left(a_{i}, t\right)\right\}
$$

holds. Hence, if $\frac{2 P}{k} \leq d_{\tilde{\ell}}\left(a_{j}, t\right)+d_{\tilde{\ell}}\left(a_{i}, t\right)$ holds for all $i \neq j$ then $f_{\tilde{\ell}}\left(a_{j}\right)=$ $\frac{k-1}{k} 2 P>P$ (for $k>2$ ) holds and otherwise $f_{\tilde{\ell}}\left(a_{j}\right) \geq f_{\tilde{\ell}}(t)$ because then there is at least one set $j^{\prime} \neq j$ with $d_{\tilde{\ell}}\left(a_{j}, a_{j^{\prime}}\right)=d_{\tilde{\ell}}\left(a_{j}, t\right)+d_{\tilde{\ell}}\left(a_{j^{\prime}}, t\right)$ and

$$
d_{\tilde{\ell}}\left(a_{j}, a_{i}\right)=\min \{\underbrace{2 P / k}_{\geq d_{\tilde{\ell}}\left(a_{i}, t\right)}, d_{\tilde{\ell}}\left(a_{j}, t\right)+d_{\tilde{\ell}}\left(a_{i}, t\right)\} \geq d_{\tilde{\ell}}\left(a_{i}, t\right)
$$

holds for all $i \neq j$. These observations immediately imply that $t$ is a 1-median with respect to $\tilde{\ell}$ if and only if $f_{\tilde{\ell}}(t) \leq P$.

Lemma 2 Assume that $I_{K P}$ is an instance of the continuous multiple choice knapsack problem. Then there exists a feasible solution $\left(\varphi^{*}, r^{*}\right)$ for $I_{K P}$ if and only if there exists a feasible solution $\left(p^{*}, q^{*}\right)$ of the corresponding instance of the inverse 1-median problem with cost at most $C$.

Proof This lemma can be proved in an analogous way as Lemma 2.3 in [10].

This lemma immediately leads to the following theorem.

Theorem 3 The inverse 1-median problem with variable edge lengths is weakly $\mathcal{N} \mathcal{P}$-hard on series-parallel graphs.

Now we consider an instance of the inverse 1-median problem on seriesparallel graphs. In an analogous way as Subsection 3.2 we construct an equivalent instance of the inverse $p$-median problem. Since the constructed graph is also a series-parallel graph we conclude the following theorem:

Theorem 4 The inverse $p$-median problem with variable edge lengths is weakly $\mathcal{N} \mathcal{P}$-hard on series-parallel graphs for every $p \in \mathbb{N}$.

\section{The inverse 2-median problem on trees}

Consider a tree graph with $n$ vertices. Throughout this section, we assume that $\ell_{e}-u_{e}^{-}>0$ hold for all $e \in E$ because otherwise the modified length $\tilde{\ell}_{e}=\ell_{e}-u_{e}^{-}=0$ would change the topology of the tree (edges with zerolengths have to be contracted).

Observe that every pair of vertices is a candidate to be a 2-median. Hence, the formulation of the inverse 2-median problem (see Section 1) has $\left(\begin{array}{l}n \\ 2\end{array}\right)=$ $\mathcal{O}\left(n^{2}\right)$ constraints that make sure that the given pair will be a 2-median and in addition there are $2 n$ bound constraints. Unfortunately, the constraints (1) are nonlinear because they involve the minimum-operator, i.e., it is not clear in advance to which facility vertices travel. Our goal is now to avoid this difficulty 
of non-linearity by making use of some properties of the 2-median problem on trees.

Let us assume that $T=(V, E)$ is an undirected tree with vertex set $V$ with $|V|=n$ and edge set $E$ with $|E|=n-1$. Suppose that the unique path from $v_{i} \in V$ to $v_{j} \in V$ is denoted by $p\left[v_{i}, v_{j}\right]$ and $d_{\ell}\left(v_{i}, v_{j}\right)$ is the length of $p\left[v_{i}, v_{j}\right]$ with respect to $\ell$. A method for finding 2-medians of a tree is to consider edge deletions [20]. Observe that whenever two vertices are fixed as facility locations, there exists exactly one edge that is not used by any vertex on its way to its closest facility. This edge is called split-edge. Conversely, whenever an edge is fixed to be a split-edge the assignment of vertices to facilities is uniquely given. Hence, the idea is to guess the split-edge: The deletion of edge $e \in E$, partitions the tree $T$ into two subtrees $T_{1}(e)$ and $T_{2}(e)$. Let $V_{1}(e)$ and $V_{2}(e)$ be the corresponding vertex sets of $T_{1}(e)$ and $T_{2}(e)$, respectively. Since we have $(n-1)$ edges we have $(n-1)$ partitions. For each edge deletion we determine, using Goldman's algorithm [13,20], a 1-median $m_{1}(e)$ in $T_{1}(e)$ and a 1-median $m_{2}(e)$ in $T_{2}(e)$. Let $\bar{f}_{\ell}(e)$ be the optimal objective value provided that $e$ is the split-edge and $\left\{m_{1}(e), m_{2}(e)\right\}$ are the facility locations. Then $\bar{f}_{\ell}(e)$ is equal to

$$
\bar{f}_{\ell}(e)=\sum_{v \in V_{1}(e)} w(v) d_{\ell}\left(v, m_{1}(e)\right)+\sum_{v \in V_{2}(e)} w(v) d_{\ell}\left(v, m_{2}(e)\right)
$$

A consequence of the edge deletion method is the following lemma (see e.g., $[20])$ :

\section{Lemma 3}

i) $f_{\ell}\left(m_{1}(e), m_{2}(e)\right) \leq \bar{f}_{\ell}(e)$ for all $e \in E$.

ii) If the set $\left\{m_{1}, m_{2}\right\}$ is a 2-median of $T$ then there exists an edge $e^{*} \in E$ such that $m_{1}$ and $m_{2}$ are 1-medians of $T_{1}\left(e^{*}\right)$ and $T_{2}\left(e^{*}\right)$, respectively, and $\bar{f}_{\ell}\left(e^{*}\right)=f_{\ell}\left(m_{1}, m_{2}\right)$.

iii) If $\bar{f}_{\ell}\left(e^{*}\right) \leq \bar{f}_{\ell}(e)$ for all $e \in E$ then the set $\left\{m_{1}\left(e^{*}\right), m_{2}\left(e^{*}\right)\right\}$ is a 2-median.

Lemma 3 says that for every fixed edge $e$ we can determine a 1-median $m_{i}(e)$ for $i=1,2$ in $T_{i}(e)$ (according to Goldman's algorithm $[13,20]$ ) and finally take the best pair among the candidates $\left\{\left\{m_{1}(e), m_{2}(e)\right\} \mid e \in E\right\}$. Observe that there exists an optimality criterion for the 1-median problem in a graph that is independent of the edge lengths (this property is often called Goldman property [13]). Since we modify only edge lengths while vertex weights are fixed, we can determine this set of candidates $\left\{\left\{m_{1}(e), m_{2}(e)\right\} \mid\right.$ $e \in E\}$ without knowledge about the final edge lengths.

Let us assume that the prespecified set $\left\{m_{1}, m_{2}\right\}$ is not a 2-median of $T$. We want to modify the edge lengths $\ell_{e}, e \in E$, to $\tilde{\ell}_{e}$ at minimum cost such that the given set $\left\{m_{1}, m_{2}\right\}$ becomes a 2 -median. The difficulty is, however, that we do not know the correct split-edge. Hence, we try out all possibilities $e \in p\left[m_{1}, m_{2}\right]$. Whenever $m_{i}$ are 1-medians in $T_{i}(e)(i=1,2)$ then $e$ is a candidate be an optimal split-edge. Observe that an edge $e \in p\left[m_{1}, m_{2}\right]$ with 
$m_{i} \in T_{i}(e)$ is not a 1-median in $T_{i}(e)$ can not be a split-edge with respect to an optimal solution of the inverse 2 -median problem.

Let $\mathcal{M}$ denote the set of split-edge candidates. For every $e^{*} \in \mathcal{M}$, we are interested in an optimal length modification such that $\bar{f}_{\tilde{\ell}}\left(e^{*}\right) \leq \bar{f}_{\tilde{\ell}}(e)$ holds for every $e \in E$. Let $e^{*} \in \mathcal{M}$ then the following linear programming problem models the problem of finding an optimal length modification with the additional constraint that $e^{*}$ is the split-edge:

$$
\begin{array}{cll}
\left(\mathrm{P}_{1}\right) \text { minimize } & \sum_{e \in E}\left(c_{e}^{+} p_{e}+c_{e}^{-} q_{e}\right) & \\
\text { subject to } & \bar{f}_{\tilde{\ell}}\left(e^{*}\right) \leq \bar{f}_{\tilde{\ell}}(e) & \text { for all } e \in E, \\
& \tilde{\ell}_{e}=\ell_{e}+p_{e}-q_{e} & \text { for all } e \in E, \\
& 0 \leq p_{e} \leq u_{e}^{+} & \text {for all } e \in E, \\
& 0 \leq q_{e} \leq u_{e}^{-} & \text {for all } e \in E
\end{array}
$$

Since

$$
d_{\tilde{\ell}}\left(v_{i}, v_{j}\right)=\sum_{e \in p\left[v_{i}, v_{j}\right]}\left(\ell_{e}+p_{e}-q_{e}\right)
$$

is linear, constraints (2) are also linear. Thus, an optimal solution of the inverse 2-median problem can be found by solving $\left(\mathrm{P}_{1}\right)$ for every $e \in \mathcal{M}$ and finally choosing a cheapest solution. This implies that the inverse 2 -median problem on a tree is solvable in polynomial time.

Note that if we allow to reduce edge lengths to zero then we also have to consider split-edges $e \in p\left[m_{1}, m_{2}\right]$ where $m_{i} \in T_{i}(e)(i=1,2)$ are not 1medians in their subtrees. In this case, we determine the cost of changing all edges on a path from $m_{i}$ to $m_{i}(e)$ to zero (i.e., we change the topology of the tree) and continue with the linear programming problem for the modified tree. The cost of edge $e \in p\left[m_{1}, m_{2}\right]$ is then given by the cost of zero-length modification plus the cost of length modification given by the linear program. Since we assume positive vertex weights, every subtree may have up to two different 1-medians. Hence, for every edge $e \in p\left[m_{1}, m_{2}\right]$, we have to solve up to four linear programming problems.

Theorem 5 The inverse 2-median problem can be solved in polynomial time if the underlying network is a tree.

\section{The inverse 2-median problem on star graphs}

Let $G=(V, E)$ be a star graph with vertex set $V=\left\{v_{0}, v_{1}, \ldots, v_{n}\right\}$ and edge set $E=\left\{e_{i}=\left(v_{0}, v_{i}\right) \mid i=1, \ldots, n\right\}, v_{0}$ is the central vertex and $v_{1}, \ldots, v_{n}$ are leaves of $G$. Assume that $w\left(v_{i}\right)=w_{i}, i=0,1, \ldots, n$ are vertex weights of $G$ and

$$
w_{n} \geq w_{n-1} \geq \ldots \geq w_{1} .
$$


The deletion of edge $e_{i} \in E$ partitions the star graph $G$ into the two subgraphs $G_{1}\left(e_{i}\right)$ and $G_{2}\left(e_{i}\right)$, where $G_{1}\left(e_{i}\right)=\left(V_{1}\left(e_{i}\right), E_{1}\left(e_{i}\right)\right)$ is the trivial graph with vertex set $V_{1}\left(e_{i}\right)=\left\{v_{i}\right\}$ and $G_{2}\left(e_{i}\right)$ is a star graph with vertex set $V_{2}\left(e_{i}\right)=$ $\left\{v_{0}, v_{1}, \ldots, v_{i-1}, v_{i+1}, \ldots, v_{n}\right\}$ and edge set $E=\left\{e_{1}, \ldots, e_{i-1}, e_{i+1}, \ldots, e_{n}\right\}$.

Our task is to compute $\bar{f}_{\tilde{\ell}}\left(e_{i}\right)$ for $i=1, \ldots, n$ in order to apply the solution method described in the previous section. Let us first compute the 1-medians $m_{1}\left(e_{i}\right)$ and $m_{2}\left(e_{i}\right)$ :

Assume that $e_{i}, i=1, \ldots, n-1$ is a split-edge in $G$. In this case $m_{1}\left(e_{i}\right)=v_{i}$ is the 1-median in $G_{1}\left(e_{i}\right)$. In order to determine a 1-median $m_{2}\left(e_{i}\right)$ in $G_{2}\left(e_{i}\right)$, we use Goldman's optimality criterion $[13,20]$ : If the weight of vertex $v_{n}$ is not large enough, i.e.,

$$
w_{n}<\sum_{j=0}^{n} w_{j}-w_{n}-w_{i}
$$

then $m_{2}\left(e_{i}\right)=v_{0}$ is a 1-median in $G_{2}\left(e_{i}\right)$, otherwise $m_{2}\left(e_{i}\right)=v_{n}$. Thus, for $i=1, \ldots, n-1$ we have

$$
\bar{f}_{\ell}\left(e_{i}\right)=\sum_{j=1}^{n} w_{j} \ell_{e_{j}}-w_{i} \ell_{e_{i}}-a_{i} \ell_{e_{n}}
$$

with

$$
a_{i}= \begin{cases}0 & \text { if } w_{n}<\sum_{j=0}^{n} w_{j}-w_{n}-w_{i} \\ 2 w_{n}+w_{i}-\sum_{j=0}^{n} w_{j} & \text { if } w_{n} \geq \sum_{j=0}^{n} w_{j}-w_{n}-w_{i} .\end{cases}
$$

Now assume that $e_{n}$ is a split-edge in $G$. In this case $m_{1}\left(e_{n}\right)=v_{n}$ holds. If the weight of vertex $v_{n-1}$ is not large enough, i.e.,

$$
w_{n-1}<\sum_{j=0}^{n} w_{j}-w_{n-1}-w_{n},
$$

then $m_{2}\left(e_{n}\right)=v_{0}$, otherwise $m_{2}\left(e_{n}\right)=v_{n-1}$. Therefore,

$$
\bar{f}_{\ell}\left(e_{n}\right)=\sum_{j=1}^{n} w_{j} \ell_{e_{j}}-w_{n} \ell_{e_{n}}-a_{n} \ell_{e_{n-1}}
$$

with

$$
a_{n}= \begin{cases}0 & \text { if } w_{n-1}<\sum_{j=0}^{n} w_{j}-w_{n-1}-w_{n} \\ 2 w_{n-1}+w_{n}-\sum_{j=0}^{n} w_{j} & \text { if } w_{n-1} \geq \sum_{j=0}^{n} w_{j}-w_{n-1}-w_{n} .\end{cases}
$$

Let now assume that we are given a set $\left\{m_{1}, m_{2}\right\}$ of vertices and we want to modify the edge lengths of $G$ at minimum cost within the given bounds such that $\left\{m_{1}, m_{2}\right\}$ becomes a 2 -median. We use the algorithm of Section 4 to compute an optimal solution of the inverse 2-median problem. Observe 
that there are at most two edges on $p\left[m_{1}, m_{2}\right]$. Hence, there are at most two candidates for an optimal split-edge. Assume that $e_{s} \in p\left[m_{1}, m_{2}\right]$ is fixed to be a split-edge of an optimal solution. The task is to solve the linear programming problem for split-edge $e_{s}$ as described in Section 4 .

Observe that every function $\bar{f}_{\ell}\left(e_{i}\right)$ for $i=1, \ldots, n$ contains the term $\sum_{j=1}^{n} w_{j} \ell_{e_{j}}$. Therefore, we can delete this term and consider the functions $g_{\ell}\left(e_{i}\right)=w_{i} \ell_{e_{i}}+a_{i} \ell_{e_{n}}$ for $i=1, \ldots, n-1$ and $g_{\ell}\left(e_{n}\right)=w_{n} \ell_{e_{n}}+a_{n} \ell_{e_{n-1}}$.

In order to simplify the explanation, we distinguish the following three cases:

Case 1. If $e_{s}=e_{n}$, then according to problem $\left(\mathrm{P}_{1}\right)$ we have to solve

$$
\begin{array}{cll}
\left(\mathrm{P}_{2}\right) \text { minimize } & \sum_{i=1}^{n}\left(c_{e_{i}}^{+} p_{e_{i}}+c_{e_{i}}^{-} q_{e_{i}}\right) & \\
\text { subject to } & w_{i} \tilde{\ell}_{e_{i}}+a_{i} \tilde{\ell}_{e_{n}} \leq w_{n} \tilde{\ell}_{e_{n}}+a_{n} \tilde{\ell}_{e_{n-1}} & \\
& \tilde{\ell}_{e_{i}}=\ell_{e_{i}}+p_{e_{i}}-q_{e_{i}} & \text { for } i=1, \ldots, n-1, \\
& 0 \leq p_{e_{i}} \leq u_{e_{i}}^{+} & \text {for } i=1, \ldots, n, \\
& 0 \leq q_{e_{i}} \leq u_{e_{i}}^{-} & \text {for } i=1, \ldots, n,
\end{array}
$$

Observe that $w_{n}-a_{i} \geq 0$ holds for all $i=1, \ldots, n-1$ and hence there exists an optimal solution with $q_{e_{n}}=0$. It is not clear in advance whether the length of $e_{n-1}$ has to be in- or decreased. Therefore, we will consider two cases where either $p_{e_{n-1}}=0$ or $q_{e_{n-1}}=0$. Finally, every $\tilde{\ell}_{e_{i}}$ for $i=1, \ldots, n-2$ appears once in constraints (3) on the left-hand side and hence $p_{e_{i}}=0$. Moreover,

$$
w_{i}\left(\ell_{e_{i}}-q_{e_{i}}\right)+a_{i} \tilde{\ell}_{e_{n}} \leq w_{n} \tilde{\ell}_{e_{n}}+a_{n} \tilde{\ell}_{e_{n-1}}=g_{\tilde{\ell}}\left(e_{n}\right)
$$

implies

$$
q_{e_{i}} \geq \ell_{e_{i}}+\frac{a_{i} \tilde{\ell}_{e_{n}}-g_{\tilde{\ell}}\left(e_{n}\right)}{w_{i}}=A_{i}\left(\tilde{\ell}_{e_{n-1}}, \tilde{\ell}_{e_{n}}\right)
$$

Obviously, there exists an optimal solution with

$$
q_{e_{i}}=\max \left\{A_{i}\left(\tilde{\ell}_{e_{n-1}}, \tilde{\ell}_{e_{n}}\right), 0\right\}=G_{i}\left(\tilde{\ell}_{e_{n-1}}, \tilde{\ell}_{e_{n}}\right) \quad \text { for } \quad i=1, \ldots, n-2,
$$

where

$$
A_{i}\left(\tilde{\ell}_{e_{n-1}}, \tilde{\ell}_{e_{n}}\right) \leq u_{e_{i}}^{-} .
$$

The idea is to replace the decision variables $q_{e_{i}}$ for $i=1, \ldots, n-2$ by the above representation which depends on $\tilde{\ell}_{e_{n}}$ and $\tilde{\ell}_{e_{n-1}}$. However, $\tilde{\ell}_{e_{n}}$ and $\tilde{\ell}_{e_{n-1}}$ only depend on $p_{e_{n}}$ and $p_{e_{n-1}}$ or $q_{e_{n-1}}$. In the following, we will discuss the case $p_{e_{n-1}}=0$. The other case where $q_{e_{n-1}}=0$ can be considered in an analogous way.

Observe that the constraint (3) for $i=n-1$ is equivalent to

$$
q_{e_{n-1}}\left(a_{n}-w_{n-1}\right)+p_{e_{n}}\left(a_{n-1}-w_{n}\right) \leq g_{\ell}\left(e_{n}\right)-g_{\ell}\left(e_{n-1}\right) .
$$

Hence, we have to solve 


$$
\begin{aligned}
\left(\mathrm{P}_{3}\right) \text { minimize } & \sum_{i=1}^{n-2} c_{e_{i}}^{-} G_{i}\left(\tilde{\ell}_{e_{n-1}}, \tilde{\ell}_{e_{n}}\right)+c_{e_{n-1}}^{-} q_{e_{n-1}}+c_{e_{n}}^{+} p_{e_{n}} \\
\text { subject to } & A_{i}\left(\tilde{\ell}_{e_{n-1}}, \tilde{\ell}_{e_{n}}\right) \leq u_{e_{i}}^{-} \quad \text { for } i=1, \ldots, n-2, \\
& q_{e_{n-1}}\left(a_{n}-w_{n-1}\right)+p_{e_{n}}\left(a_{n-1}-w_{n}\right) \leq g_{\ell}\left(e_{n}\right)-g_{\ell}\left(e_{n-1}\right) \\
& 0 \leq q_{e_{n-1}} \leq u_{e_{n-1}}^{-} \\
& 0 \leq p_{e_{n}} \leq u_{e_{n}}^{+} .
\end{aligned}
$$

We now explain how to solve optimization problems of the above form.

Note that $G_{i}\left(\tilde{\ell}_{e_{n-1}}, \tilde{\ell}_{e_{n}}\right)$ is a convex piecewise linear function depending on $p_{e_{n}}$ and $q_{e_{n-1}}$. If it is known whether

$$
G_{i}\left(\tilde{\ell}_{e_{n-1}}, \tilde{\ell}_{e_{n}}\right)=0 \text { or } G_{i}\left(\tilde{\ell}_{e_{n-1}}, \tilde{\ell}_{e_{n}}\right)=A_{i}\left(\tilde{\ell}_{e_{n-1}}, \tilde{\ell}_{e_{n}}\right)
$$

then objective function of problem $\left(\mathrm{P}_{3}\right)$ is linear. In other words, problem $\left(\mathrm{P}_{3}\right)$ is then a linear programming problem with $n+1$ constraints and two variables which is solvable in linear time [19].

Consider the $\left(q_{e_{n-1}}, p_{e_{n}}\right)$-plane. Then $A_{i}\left(\tilde{\ell}_{e_{n-1}}, \tilde{\ell}_{e_{n}}\right)=0$ defines a line $\mathrm{E}_{i}$ that splits the plane into two regions such that $G_{i}\left(\tilde{\ell}_{e_{n-1}}, \tilde{\ell}_{e_{n}}\right)=A_{i}\left(\tilde{\ell}_{e_{n-1}}, \tilde{\ell}_{e_{n}}\right)$ in one region while $G_{i}\left(\tilde{\ell}_{e_{n-1}}, \tilde{\ell}_{e_{n}}\right)=0$ in the other region. Hence, the task is to find the relative position of an optimal solution to these lines. Moreover, the set of points that satisfy a constraint with equality is a line, i.e., every constraint defines a line, say $\mathcal{C}_{j}$ for the $j$ 'th constraint. Hence, we are given $2 n-3=\mathcal{O}(n)$ lines in the plane.

In order to find out the relative position of an optimal solution to the lines $\mathcal{L}_{i}$, we make use of the linear search algorithm in fixed dimension due to Megiddo [19]. The key point of Megiddo's search algorithm is to find in linear time (in terms of the number of active lines) a quarter of the plane that contains an optimal solution such that a fixed fraction of lines (of type $\mathcal{L}_{i}$ or $\mathcal{C}_{j}$ ) do not intersect the quarter and hence can be discarded, i.e., they are inactive and not considered any more (in case of a line $\mathcal{L}_{i}$ the corresponding linear term is then cumulated). Megiddo provides a linear time algorithm provided that the following subproblem can be solved in linear time: Let $K$ be a line and let $\mathcal{H}_{L}$ and $\mathcal{H}_{R}$ be the two (open) halfplanes defined by $K$. If there exists an optimal solution on $K$ then determine it. Otherwise, compute the halfplane $\mathcal{H}_{L}$ or $\mathcal{H}_{R}$ that contains an optimal solution.

Now we explain the main ideas how to solve this subproblem. Clearly, if there is no feasible solution in one of the halfplanes then the answer is trivial. Now assume that there are feasible solutions in $\mathcal{H}_{L}$ and in $\mathcal{H}_{R}$. We minimize the objective function restricted to $K$ where $P$ denotes a minimal point on $K$ if no constraints are considered and $P^{\prime}$ denotes a minimal point on $K$ if the constraints are considered. Observe that both points $P^{\prime}$ and $P$ (if the problem is not bounded) can be found in linear time by considering the intersection points of the lines $\mathcal{L}_{i}$ and $\mathcal{C}_{j}$ with $K$. 
Assume that $P$ exists and lies in the interior of the feasible region. Then the slopes of the supporting planes of the objective function in $P$ enable the decision whether there is an optimal solution in $\mathcal{H}_{L}$ or in $\mathcal{H}_{R}$. If $P$ does not exist, lies outside or on the boundary of the feasible region then $P^{\prime}$ also lies on the boundary and hence $P^{\prime}$ lies on a line $\mathcal{C}_{j}$. Then compute a minimum feasible solution restricted to $\mathcal{C}_{j}$. If this solution lies in $\mathcal{H}_{L}$ (and $\mathcal{H}_{R}$, resp.) then there exists an optimal solution in $\mathcal{H}_{L}$ (an $\mathcal{H}_{R}$, resp.). Otherwise, $P^{\prime}$ is a minimum feasible solution restricted to $\mathcal{C}_{j}$. If $P$ exists then the slopes of the supporting planes of the objective function in $P$ enable the decision whether a minimum point (without considering the constraints) lies in $\mathcal{H}_{L}$ (and $\mathcal{H}_{R}$, resp.) and due to the convexity of the objective function there also exists an optimal feasible solution in $\mathcal{H}_{L}$ (and $\mathcal{H}_{R}$, resp.). If, however, $P$ does not exist, then the convexity of the objective function implies that $P^{\prime}$ is an optimal solution which lies on $K$.

Observe that the procedure described above takes linear time in the number of active lines $\mathcal{L}_{i}$ and $\mathcal{C}_{j}$ and hence Megiddo's search algorithm immediately implies a linear time algorithm for the whole problem.

Case 2. If $e_{s} \notin\left\{e_{n}, e_{n-1}\right\}$, then we can formulate the inverse 2-median problem on the star graph $G$ as follows:

$$
\begin{aligned}
& \left(\mathrm{P}_{4}\right) \text { minimize } \sum_{i=1}^{n}\left(c_{e_{i}}^{+} p_{e_{i}}+c_{e_{i}}^{-} q_{e_{i}}\right) \\
& \text { subject to } w_{i} \tilde{\ell}_{e_{i}}+a_{i} \tilde{\ell}_{e_{n}} \leq w_{s} \tilde{\ell}_{e_{s}}+a_{s} \tilde{\ell}_{e_{n}} \quad \text { for } i=1, \ldots, n-1, i \neq s \text {, } \\
& w_{n} \tilde{\ell}_{e_{n}}+a_{n} \tilde{\ell}_{e_{n-1}} \leq w_{s} \tilde{\ell}_{e_{s}}+a_{s} \tilde{\ell}_{e_{n}} \\
& \tilde{\ell}_{e_{i}}=\ell_{e_{i}}+p_{e_{i}}-q_{e_{i}} \quad \text { for } i=1, \ldots, n \text {, } \\
& 0 \leq p_{e_{i}} \leq u_{e_{i}}^{+} \quad \text { for } i=1, \ldots, n \text {, } \\
& 0 \leq q_{e_{i}} \leq u_{e_{i}}^{-} \quad \text { for } i=1, \ldots, n \text {. }
\end{aligned}
$$

The reason why we consider this case separately is that there are only $n-3$ lengths that appear in only one constraint. Let us first fix the directions of modifications: It is easy to see that there exists an optimal solution with $q_{e_{s}}=0$ and $p_{e_{i}}=0$ for $i=1, \ldots, s-1, s+1, \ldots, n-1$. Unfortunately, we do not know whether the length of $e_{n}$ is increased or decreased therefore we consider both possibilities, i.e., we solve the problem for $p_{e_{n}}=0$ and for $q_{e_{n}}=0$.

For $i=1, \ldots, n-2$ and $i \neq s$, we have $q_{e_{i}}=\max \left\{A_{i}\left(\tilde{\ell}_{e_{s}}, \tilde{\ell}_{e_{n}}\right), 0\right\}$ and $A_{i}\left(\tilde{\ell}_{e_{s}}, \tilde{\ell}_{e_{n}}\right) \leq u_{e_{i}}^{-}$. Moreover, the constraints (4) and (5) that involve $q_{e_{n-1}}$ are of the form:

$$
\begin{gathered}
w_{n-1} \tilde{\ell}_{e_{n-1}}+a_{n-1} \tilde{\ell}_{e_{n}} \leq w_{s} \tilde{\ell}_{e_{s}}+a_{s} \tilde{\ell}_{e_{n}} \\
w_{n} \tilde{\ell}_{e_{n}}+a_{n} \tilde{\ell}_{e_{n-1}} \leq w_{s} \tilde{\ell}_{e_{s}}+a_{s} \tilde{\ell}_{e_{n}}
\end{gathered}
$$


Inequality (6) implies that

$$
q_{e_{n-1}} \geq \ell_{e_{n-1}}+\frac{a_{n-1} \tilde{\ell}_{e_{n}}-g_{\tilde{\ell}}\left(e_{s}\right)}{w_{n-1}}=B_{n-1}\left(\tilde{\ell}_{e_{s}}, \tilde{\ell}_{e_{n}}\right)
$$

and from inequality (7) we get

$$
q_{e_{n-1}} \geq \ell_{e_{n-1}}+\frac{w_{n} \tilde{\ell}_{e_{n}}-g_{\tilde{\ell}}\left(e_{s}\right)}{a_{n}}=A_{n-1}\left(\tilde{\ell}_{e_{s}}, \tilde{\ell}_{e_{n}}\right) .
$$

There exists an optimal solution that satisfies one of the following two subcases: Either

$$
q_{e_{n-1}}=\max \left\{A_{n-1}\left(\tilde{\ell}_{e_{s}}, \tilde{\ell}_{e_{n}}\right), 0\right\}=G_{n-1}\left(\tilde{\ell}_{e_{s}}, \tilde{\ell}_{e_{n}}\right)
$$

with $B_{n-1}\left(\tilde{\ell}_{e_{s}}, \tilde{\ell}_{e_{n}}\right) \leq A_{n-1}\left(\tilde{\ell}_{e_{s}}, \tilde{\ell}_{e_{n}}\right)$ and $A_{n-1}\left(\tilde{\ell}_{e_{s}}, \tilde{\ell}_{e_{n}}\right) \leq u_{e_{n-1}}^{-}$or

$$
q_{e_{n-1}}=\max \left\{B_{n-1}\left(\tilde{\ell}_{e_{s}}, \tilde{\ell}_{e_{n}}\right), 0\right\}=G_{n-1}\left(\tilde{\ell}_{e_{s}}, \tilde{\ell}_{e_{n}}\right)
$$

with $A_{n-1}\left(\tilde{\ell}_{e_{s}}, \tilde{\ell}_{e_{n}}\right) \leq B_{n-1}\left(\tilde{\ell}_{e_{s}}, \tilde{\ell}_{e_{n}}\right)$ and $B_{n-1}\left(\tilde{\ell}_{e_{s}}, \tilde{\ell}_{e_{n}}\right) \leq u_{e_{n-1}}^{-}$.

The first alternative leads to the following linear programming problem (the second alternative leads to an analogue problem which we do not state explicitly):

$$
\begin{aligned}
\left(\mathrm{P}_{5}\right) \operatorname{minimize} & \sum_{\substack{i=1 \\
i \neq s}}^{n-1} c_{e_{i}}^{-} G_{i}\left(\tilde{\ell}_{e_{s}}, \tilde{\ell}_{e_{n}}\right)+c_{e_{s}}^{+} p_{e_{s}}+c_{e_{n}}^{+} p_{e_{n}}+c_{e_{n}}^{-} q_{e_{n}} \\
\text { subject to } & A_{i}\left(\tilde{\ell}_{e_{s}}, \tilde{\ell}_{e_{n}}\right) \leq u_{e_{i}}^{-} \quad \text { for } i=1, \ldots, n-1, i \neq s, \\
& B_{n-1}\left(\tilde{\ell}_{e_{s}}, \tilde{\ell}_{e_{n}}\right) \leq A_{n-1}\left(\tilde{\ell}_{e_{s}}, \tilde{\ell}_{e_{n}}\right), \\
& 0 \leq p_{e_{s}} \leq u_{e_{s}}^{+}, \\
& 0 \leq p_{e_{n}} \leq u_{e_{n}}^{+} \\
& 0 \leq q_{e_{n}} \leq u_{e_{n}}^{-} .
\end{aligned}
$$

If we fix $p_{e_{n}}=0$ or $q_{e_{n}}=0$ the resulting problem is of the same form as discussed in Case 1. Hence, we may apply Megiddo's multidimensional search and afterwards solve a linear programming problem in two variables which again leads to a linear time algorithm for the inverse 2-median problem.

Case 3. If $e_{s}=e_{n-1}$ then the inverse 2-median problem can be formulated as problem $\left(\mathrm{P}_{4}\right)$. In this case we compute $q_{e_{i}}$ for $i=1, . ., n-2$ from constraints (4) in terms of $\tilde{\ell}_{e_{n-1}}$ and $\tilde{\ell}_{e_{n}}$ and replace them in objective function of problem $\left(\mathrm{P}_{4}\right)$. Finally, we get an optimization problem with maximum operations in the objective function and $n+1$ constraints with two variables $\tilde{\ell}_{e_{n-1}}$ and $\tilde{\ell}_{e_{n}}$ which is solvable in linear time.

Now based on all cases discussed above we conclude the following theorem.

Theorem 6 The inverse 2-median problem with variable edge lengths on star graphs can be solved in linear time. 


\section{Conclusions}

We have shown that the inverse $p$-median problem with variable edge lengths on general graphs is strongly $\mathcal{N} \mathcal{P}$-hard and remains weakly $\mathcal{N} \mathcal{P}$-hard on seriesparallel graphs. However, this problem is solvable in polynomial time for $p=2$ if the underlying graph is a tree. Our approach can easily be adapted for arbitrary values of $p$. In this case the resulting linear programming problem would have $\left(\begin{array}{c}n \\ p-1\end{array}\right)$ constraints. Moreover, we developed fast algorithms for the inverse 2-median problem on a star.

The area of inverse $p$-median problems still offers a lot of open questions: It seems to be a challenging task to develop efficient algorithms for other special trees like binary trees or paths.

Moreover, inverse $p$-median problems with variable vertex weights can be solved in polynomial time provided that $p$ is fixed (Burkard, Pleschiutschnig, Zhang [6]). However, there are no efficient algorithms known for special graph classes if $p \geq 2$.

Acknowledgements This research is partially supported by the Austrian Science Fund (FWF) Project P18918-N18.

\section{References}

1. Alizadeh, B., R.E. Burkard, Combinatorial algorithms for inverse absolute and vertex 1-center location problems on trees. Technical Report 2009-09, Graz University of Technology (2009).

2. Alizadeh, B., R.E. Burkard, U. Pferschy, Inverse 1-center location problems with edge length augmentation on trees. Technical Report 2009-07, Graz University of Technology (2009).

3. Baroughi Bonab, F., R.E. Burkard, B. Alizadeh, Inverse median location problems with variable coordinates. Technical Report 2009-8, Graz University of Technology (2009).

4. Benkoczi, R., B. Bhattacharya, A new template for solving $p$-median problems for trees in sub-quadratic time (extended abstract). Lecture Notes in Comput. Sci. 3669, 271-282 (2005).

5. Burkard, R.E., M. Galavii, E. Gassner, The inverse Fermat-Weber problem. Technical Report 2008-14, Graz University of Technology (2008).

6. Burkard, R.E., C. Pleschiutschnig, J. Zhang, Inverse median problems. Discrete Optimization 1, 23-39 (2004).

7. Burkard, R.E., C. Pleschiutschnig, J.Z. Zhang, The inverse 1- median problem on a cycle. Discrete Optimization 5, 242-253 (2008).

8. Burton, D., Ph.L. Toint, On an instance of the inverse shortest path problem. Mathematical Programming 53, 45-61 (1992).

9. Cai, M.C., X.G. Yang, J.Z. Zhang, The complexity analysis of the inverse center location problem. Journal of Global Optimization 15, 213-218 (1999).

10. Gassner, E., The inverse 1-maxian problem with edge length modification. Journal of Combinatorial Optimization 16, 50-67 (2008).

11. Gassner, E., An inverse approach to convex ordered median problems in trees. Technical Report 2008-16, Graz University of Technology (2008).

12. Garey, M.R., D.S. Johnson, Computers and intractability: a guide to the theory of NPcompleteness. W.H. Freeman, New York (1979).

13. Goldman, A.J., Optimal center location in simple networks. Transp. Sci. 5, 212-221 (1971). 
14. Gavish, B., S. Sridhar, Computing the 2-median on tree networks in $O(n \log n)$ time. Networks 26, 305-317 (1995).

15. Hakimi, S.L., Optimum location of switching centers and the absolute centers and medians of a graph. Operations Research 12, 450-459 (1964).

16. Heuberger, C., Inverse optimization: a survey on problems, methods, and results. Journal of Combinatorial Optimization 8, 329-361 (2004).

17. Hua, L.K., et al., Applications of mathematical models to wheat harvesting. Chinese Mathematics 2, 77-91 (1962).

18. Kariv, O., L. Hakimi, An algorithmic approach to network location problems part 2: The $p$-medians. SIAM Journal of Applied Mathematics 37, 539-560 (1979).

19. Megiddo, N., Linear programming in linear time when the dimension is fixed. J. $A C M$ 31, 114-127 (1984).

20. Mirchandani, P.B., R.L. Francis, Discrete location theory. John Wiley \& Sons, New York (1990)

21. Tamir, A., An $O\left(p n^{2}\right)$ algorithm for the $p$-median and related problems on tree graphs. Operations Research Letters 19, 59-64 (1996).

22. Yang, X., J. Zhang, Inverse center location problem on a tree. Journal of Systems Science and Complexity 21, 651-664 (2008). 